\title{
Tbx3: another important piece fitted into the pluripotent stem cell puzzle
}

Melinda K Pirity ${ }^{\top}$ and Andras Dinnyes*1,2

\begin{abstract}
Induced pluripotent stem cells (iPSCs) are novel tools for biomedical research, with a promise for future regenerative medicine applications. Recently, Han and colleagues reported in Nature that T box gene 3 (Tbx3) can improve the quality of mouse iPSCs and increase their germline transmission efficacy. This observation contributes greatly to the improvement of iPSC technology and might be a step towards 'designer' reprogramming strategies by generating high quality iPSCs. Further studies comparing pluripotency regulation in different species, including that in human, will be necessary to verify the universal role of Tbx3 and the medical relevance of the observation.
\end{abstract}

Genetic reprogramming of adult somatic cells into stem cells, or other cell types, is a long-time dream for regenerative medicine. The birth of Dolly, the first reprogrammed adult cell-derived mammal, heralded a new era [1], yet more than a decade of research towards 'therapeutic cloning' has resulted in only limited progress, due to many technical, ethical and legislative problems. Recently, genetic reprogramming of cells using reprogramming factors in mouse and human [2] resulted in the production of induced pluripotent stem cells (iPSCs) and immediately became one of the most prolific and 'hot' areas of biomedical research. The generation of human iPSCs holds great promise for novel drug testing using patient-derived in vitro systems and the production of regenerative cells for tissue or organ repair, but without the ethical burden of the nuclear transfer cloning-based reprogramming methods. Nevertheless, the iPSC technology is still in its infancy, with numerous issues to be solved - a real puzzle for which many

*Correspondence: andrasdinnyes@yahoo.com

${ }^{2}$ Molecular Animal Biotechnology Laboratory, Szent Istvan University, Pater K. u. 1 , Godollo, H-2100, Hungary

Full list of author information is available at the end of the article excellent and very competitive players are currently trying to find the key fitting pieces.

Colonization of the germ layer by mouse embryonic stem cells ('germline transmission') is essential for the production of germline competent knockout and transgenic mice. Recently, Han and colleagues [3] described in Nature that the T box gene $3(T b \times 3)$ [4] is amongst the genes that can greatly improve the quality of reprogrammed iPSCs and the efficacy of germline transmission. T-box family genes encode transcription factors involved in the regulation of both embryonic and extraembryonic development of the embryo proper, especially as they are required for early cell-fate decisions, such as those necessary for formation of the basic vertebrate body plan and those involved in mesoderm formation at the early, inductive stage of development (mammary gland, lung, heart, limbs) [5]. The family is characterized by a highly conserved sequence encoding a DNA-binding domain, called the T-domain, that binds specific sequences in the promoters of target genes, originally found in the Brachyury locus. Surprisingly, although the developmental role of T-box genes is quite well described, little is known about how these proteins regulate their target genes. The results obtained by Han and colleagues not only provide some missing pieces on the role of Tbx3 during reprogramming but are also a step towards realizing 'designer' reprogramming strategies that require high quality iPSCs.

Some earlier observations that overexpression of Tbx3 promotes LIF-independent self-renewal of stem cells [6], and that a parallel LIF signaling pathway might maintain the pluripotency of mouse stem cells, have also suggested that reprogramming is a complex process, and Tbx3 may have some specific roles in this process. Researchers have also suggested before that while Klf4 is mainly activated by the Jak-Stat3 pathway and signals downstream to Sox 2 , Tbx 3 is primarily regulated by the phosphatidylinositol-3-OH kinase-Akt and mitogen-activated protein kinase pathways and predominantly stimulates Nanog [7]. Importantly, in the absence of LIF, artificial expression of Klf4 or Tbx3 is sufficient to maintain pluripotency [8]. These and other observations (for example, that Tbx3 is specifically expressed in precursor and progenitor cells, 
such as multipotent hepatic progenitor cells [9]) underscore that Tbx3 may well participate in LIF-independent or parallel pathways of pluripotency/reprogramming. These observations give new hope for transgenic research and will contribute to the generation of better transgenic and cell-based models for studying pluripotency and cell fate decision-making.

At the same time the precise relationships between other factors - for example, Fgf10, Nrg3, Wnts and their relationship to Tbx3, LIF, Oct4 or Nanog - have yet to be fully elucidated. As applications in human medicine are the main goal, it is important to emphasize that pluripotency regulation in mouse and human differs in many details - the role of each of the above factors in reprogramming needs to be clarified in different, nonrodent model species and, most importantly, in human. The search for molecular signatures of 'stemness' and pluripotency is only now beginning to gather momentum.

As many pieces of the 'pluripotency regulation puzzle' are still missing, the field will probably supply us with numerous exciting publications and - after sorting out major issues such as stability and safety of iPSCs and iPSC-derived cells - hopefully with everyday medical applications as well. But this is still several years ahead, even according to the most optimistic projections - still plenty of time for young researchers to make an exciting career in the pluripotent stem cell and genetic reprogramming area.

Abbreviations

iPSC = induced pluripotent stem cell.

\section{Competing interests}

The authors declare that they have no competing interests.

\section{Author details}

'Biotalentum Ltd, Godollo, H-2100, Hungary. ${ }^{2}$ Molecular Animal Biotechnology Laboratory, Szent Istvan University, Pater K. u. 1, Godollo, H-2100, Hungary.

\section{Acknowledgments}

MKP and AD are supported by EU FP7 (InduHeart, PEOPLE-IRG-2008-234390; PluriSys, HEALTH-2007-B-223485; PartnErS, PIAPGA-2008-218205), and NKTH/ KPI (NKTH-OTKA FP7 "Mobility" HUMAN-MB08C-80205).

Published: 20 May 2010

References

1. Wilmut I, Sullivan G, Taylor J: A decade of progress since the birth of Dolly. Reprod Fertil Dev 2009, 21:95-100.

2. Takahashi K, Yamanaka S: Induction of pluripotent stem cells from mouse embryonic and adult fibroblast cultures by defined factors. Cell 2006, 126:663-676.

3. Han J, Yuan P, Yang H, Zhang J, Soh BS, Li P, Lim SL, Cao S, Tay J, Orlov YL, Lufkin T, Ng HH, Tam WL, Lim B: Tbx3 improves the germ-line competency of induced pluripotent stem cells. Nature, 463:1096-1100.

4. Bollag RJ, Siegfried Z, Cebra-Thomas JA, Garvey N, Davison EM, Silver LM: An ancient family of embryonically expressed mouse genes sharing a conserved protein motif with the T locus. Nat Genet 1994, 7:383-389.

5. Naiche LA, Harrelson Z, Kelly RG, Papaioannou VE: T-box genes in vertebrate development. Annu Rev Genet 2005, 39:219-239.

6. Niwa H, Ogawa K, Shimosato D, Adachi K: A parallel circuit of LIF signalling pathways maintains pluripotency of mouse ES cells. Nature 2009, 460:118-122.

7. Niwa $\mathrm{H}$ : [Transcription factor network governing cellular pluripotency] Rinsho Ketsueki 2009, 50:1524-1530.

8. Savarese F, Dávila A, Nechanitzky R, De La Rosa-Velazquez I, Pereira CF, Engelke R, Takahashi K, Jenuwein T, Kohwi-Shigematsu T, Fisher AG, Grosschedl R: Satb1 and Satb2 regulate embryonic stem cell differentiation and Nanog expression. Genes Dev 2009, 23:2625-2638.

9. Suzuki A, Sekiya S, Buscher D, Izpisua Belmonte JC, Taniguchi H: Tbx3 controls the fate of hepatic progenitor cells in liver development by suppressing p19ARF expression. Development 2008, 135:1589-1595.

doi:10.1186/scrt12

Cite this article as: Pirity MK, Dinnyes A: Tbx3: another important piece fitted into the pluripotent stem cell puzzle. Stem Cell Research \& Therapy 2010, $1: 12$. 